\title{
Effect of combined treatment with micelle-incorporated cisplatin (NC-6004) and S-1 on human gastric cancer xenografts
}

\author{
MASAHISA KUDO ${ }^{1,2}$, YOSHIYUKI YAMAMOTO ${ }^{1}$, YOSHIKATSU KOGA ${ }^{1}$, TETSUYA HAMAGUCHI $^{3}$, \\ TETSUO AKIMOTO $^{2}$, MASAHIRO YASUNAGA ${ }^{1}$ and YASUHIRO MATSUMURA ${ }^{1}$ \\ ${ }^{1}$ Division of Developmental Therapeutics, Exploratory Oncology Research and Clinical Trial Center, National Cancer Center, \\ Kashiwa, Chiba 277-8577; ${ }^{2}$ Course of Advanced Clinical Research of Cancer, Juntendo University Graduate School \\ of Medicine, Tokyo 113-8421; ${ }^{3}$ Department of Gastrointestinal Medical Oncology, \\ National Cancer Center Hospital, Tokyo 104-0045, Japan
}

Received June 15, 2016; Accepted September 2, 2016

DOI: $10.3892 / \operatorname{mco} .2016 .1070$

\begin{abstract}
Combination therapy with S-1 and cisplatin (CDDP) is the standard chemotherapy for advanced gastric cancer in Japan; however, its administration requires hospitalization for hydration to prevent nephrotoxicity from CDDP. By contrast, NC-6004 appears to reduce the renal toxicity of CDDP and may be used on an outpatient basis. Thus, the effects of combined treatment with S-1 and NC-6004 were compared with those of S-1 and CDDP in a human gastric cancer model. In vitro cytotoxic effects were investigated in 44As3Luc, MKN45 and MKN74 human gastric cancer cell lines. The effects of NC-6004 and 5-fluorouracil (5-FU) were compared with the effects of CDDP and 5-FU using the combination index method. The in vivo antitumor effects of S-1/NC-6004 and S-1/CDDP were evaluated in mice bearing 44As3Luc xenografts. Both combinations exhibited synergistic activity in MKN45 and MKN74 cells and additive effects in 44As3Luc cells. Moreover, the in vivo antitumor effects did not differ between the S-1/NC-6004 and S-1/CDDP treatment groups. However, a significantly lower body weight loss was observed in S-1/NC-6004-treated mice compared with the S-1/CDDP-treated mice. Our data warrant a clinical evaluation of S-1/NC-6004 combination therapy.
\end{abstract}

Correspondence to: Dr Yasuhiro Matsumura, Division of Developmental Therapeutics, Exploratory Oncology Research and Clinical Trial Center, National Cancer Center, 6-5-1 Kashiwanoha, Kashiwa, Chiba 277-8577, Japan

E-mail: yhmatsum@east.ncc.go.jp

Abbreviations: CDDP, cisplatin; 5-FU, 5-fluorouracil; EPR, enhanced permeability and retention; GI, gastrointestinal; $\mathrm{IC}_{50}$, $50 \%$ inhibitory concentration; CI, combination index; Fa, fraction affected; TV, tumor volume; SD, standard deviation

Key words: gastric cancer, NC-6004, S-1, drug delivery system, enhanced permeability and retention effect

\section{Introduction}

In Japan, the standard chemotherapy for advanced or recurrent gastric cancer is a combination of S-1 and CDDP. However, in a phase III clinical trial (1), the median overall survival following combination therapy was only 13 months, indicating a poor prognosis for patients with advanced gastric cancer. Furthermore, administration of this combination therapy requires hospitalization for hydration to prevent nephrotoxicity from CDDP (2) and is associated with adverse effects, including severe nausea, neurotoxicity and allergic reactions (3). Due to these adverse effects, discontinuation of CDDP treatment is often necessary. As an alternative, combination therapy with S-1 and oxaliplatin is considered standard for advanced gastric cancer (4) and it may be administered on an outpatient basis, as no hydration is required with oxaliplatin administration. However, peripheral neuropathy is a major adverse effect of oxaliplatin and often warrants discontinuation of treatment due to the compromised quality of life.

Drug delivery systems include active and passive targeting approaches. Passive targeting may be achieved through the enhanced permeability and retention (EPR) effect (5), which leads to selective accumulation of micelle-incorporated anticancer agents in tumors and prominent antitumor effects, while decreasing toxicity of the drug payload (6). NC-6004, which contains CDDP-incorporating polymeric micelles, was developed to reduce the adverse effects of CDDP and enhance its antitumor activity (7). We previously reported the improved antitumor effects and reduced toxicity, including reduced nephrotoxicity, of NC-6004 in vivo (8). Peak urinary concentrations of CDDP correlate better with toxicity compared with total renal platinum concentrations (9); hence, CDDP nephrotoxicity is considered to be dependent on peak urinary concentrations and maximum CDDP concentrations in the uriniferous tubules. Therefore, strategies that allow gradual, rather than sudden, proximal and distal renal tubular CDDP accumulation may ameliorate nephrotoxicity. NC-6004 requires no concomitant medications or hydration and was shown to eliminate CDDP toxicity without attenuating the antitumor effect (10). A phase I study of NC-6004 in patients with advanced solid tumors demonstrated that delayed and 
sustained release of CDDP following intravenous administration significantly reduced the renal toxicity of NC-6004. In addition, gastrointestinal (GI) toxicity was significantly reduced, as almost all patients in the phase I trial only experienced grade 1 or weak GI toxicity (11).

The aim of the present study was to compare the effects of combined treatment with NC-6004/S-1 with the effects of CDDP/S-1 treatment in a human gastric cancer model.

\section{Materials and methods}

Chemotherapeutic agents. NC-6004, which consists of polyethylene glycol, a hydrophilic chain constituting the outer shell of micelles, and the coordinate complex of poly (glutamic acid) and CDDP (12), was prepared by NanoCarrier Co., Ltd. (Kashiwa, Japan). CDDP was purchased from Nippon Kayaku Co., Ltd. (Tokyo, Japan). S-1 was purchased from Taiho Pharmaceutical Co., Ltd. (Tokyo, Japan). 5-FU was purchased from Kyowa Hakko Kirin Co., Ltd. (Tokyo, Japan).

Cell culture. The 44As3Luc human signet ring cell gastric cancer cell line, which stably expresses firefly luciferase (12), was kindly provided by Dr K. Yanagihara (National Cancer Center Hospital East, Kashiwa, Japan). MKN45 and MKN74 cells were purchased from the JCRB Cell Bank (Osaka, Japan). The cell lines were maintained in RPMI-1640 medium (Wako, Osaka, Japan) containing 10\% fetal bovine serum (Roche Diagnostics, Tokyo, Japan), $100 \mathrm{U} / \mathrm{ml}$ penicillin, $100 \mu \mathrm{g} / \mathrm{ml}$ streptomycin and $25 \mu \mathrm{g} / \mathrm{ml}$ amphotericin B (Wako) in a humidified atmosphere containing $5 \% \mathrm{CO}_{2}$ at $37^{\circ} \mathrm{C}$.

In vitro growth inhibition assays. The growth inhibitory effects of NC-6004, CDDP and 5-FU were investigated using tetrazolium salt-based proliferation assays (WST-8 assay; Wako). In these in vitro studies, 5 -FU was used instead of S-1, as tegafur is a fluorouracil prodrug that is mainly activated in the liver. S-1 consists of tegafur, two modulators of 5-FU metabolism, 5-chloro-2,4-dihydroxypyridine (CDHP), a reversible inhibitor of dihydropyrimidine dehydrogenase (DPD), and potassium oxonate, in a molar ratio of 1: 0.4:1. Tegafur, an oral prodrug of 5-FU, is gradually converted to 5-FU and rapidly metabolised by DPD in the liver. CDHP exhibits a DPD-inhibitory activity that is 180 -fold higher compared with that of uracil, and was confirmed to be an effective DPD inhibitor in the form of uracil/tegafur (UFT) in vitro. Potassium oxonate is an orotate phosphoribosyl transferase inhibitor that is distributed primarily to the gastrointestinal tract. This component of S-1 decreases the incorporation of 5-fluorouridine triphosphate into RNA in the gastrointestinal mucosa and reduces the incidence of diarrhea (13). The 44As3Luc, MKN45 and MKN74 cells were seeded into 96 -well plates at 3,000 cells/well in a final volume of $100 \mu \mathrm{l}$, and were incubated for $24 \mathrm{~h}$ at $37^{\circ} \mathrm{C}$. Following removal of the media, 100- $\mu 1$ aliquots of medium containing various concentrations of each drug were added to the wells, and the cells were incubated for $72 \mathrm{~h}$ at $37^{\circ} \mathrm{C}$. After removing the media, $10 \mu \mathrm{l}$ of WST- 8 solution and $90 \mu \mathrm{l}$ of media were added to wells, and the cells were incubated for another $3 \mathrm{~h}$ at $37^{\circ} \mathrm{C}$. Subsequently, absorbance of the formazan product was detected at $450 \mathrm{~nm}$ using a 96 -well spectrophotometric plate reader (SpectraMax 190, Molecular
Devices Corp., Sunnyvale, CA, USA). The experiments were performed in triplicate and repeated at least three times. Cell viability data are expressed as means and were normalized to data from non-treated control cells to generate dose-response curves. Finally, the 50\% inhibitory concentrations $\left(\mathrm{IC}_{50}\right)$ were determined from the dose-response curves.

Drug interaction analyses. Interactions between NC-6004 or CDDP and 5-FU in gastric cancer cell lines were evaluated according to the combination index (CI) method described by Chou and Talalay (14). The data were analyzed using Calcusyn software (Biosoft, New York, NY, USA) and 5-FU was combined with NC-6004 or CDDP at fixed ratios spanning the individual $\mathrm{IC}_{50}$ values for each drug. For any given drug combination, the $\mathrm{CI}$ values represent degrees of synergy and antagonism, and are expressed in terms of the fraction affected $(\mathrm{Fa})$ values, which represent percentages of cells killed or inhibited by the drug. $\mathrm{Fa} / \mathrm{CI}$ plots were constructed using computer analyses of data generated from median effect analyses.

Experimental mouse models. A total 150 6-week-old female $\mathrm{BALB} / \mathrm{c}$ nude mice, weighing $\sim 16 \mathrm{~g}$, were purchased from SLC Japan (Shizuoka, Japan). The mice were housed in cages under specific pathogen-free conditions and were provided free access to sterile food and water. Seven-week-old mice were subcutaneously inoculated in the flank with $1 \times 10^{6} 44$ As3 Luc cells. All animal procedures were performed in compliance with the guidelines for the care and use of experimental animals established by the Committee for Animal Experimentation of the National Cancer Center. These guidelines meet the ethical standards required by law and comply with the guidelines for the use of experimental animals in Japan.

In vivo growth inhibition assays. The tumors were grown to a volume of $70 \mathrm{~mm}^{3}$, and the mice were randomly divided into test groups of 4 or 6 mice ( 4 for the control and 6 for the treatment group) (day 0). Subsequently, NC-6004 and CDDP were administered intravenously via the lateral tail veins, whereas S-1 was administered orally. The normal control group received a $0.9 \% \mathrm{NaCl}$ solution. The body weight and body length (a) and the width of the tumor mass (b) was measured twice weekly. The tumor volume (TV) was calculated using the following equation: $\mathrm{TV}=\left(\mathrm{axb}^{2}\right) / 2$. For humane reasons, animals with TV $>2,000 \mathrm{~mm}^{3}$ were sacrificed.

We initially evaluated the antitumor effects of NC-6004, CDDP, or S-1 individually. NC-6004 (an equivalent dose of CDDP) and CDDP were administered at doses of 2.5, 5.0 and $7.5 \mathrm{mg} / \mathrm{kg}$, and S-1 was administered at doses of 5.0, 10.0, and $15.0 \mathrm{mg} / \mathrm{kg}$. NC-6004 and CDDP were administered on days 1 and 8 , and S-1 was administered on days $0-4$ and 7-11. The antitumor effects of NC-6004/S-1 or CDDP/S-1 were evaluated following administration of $2.5 \mathrm{mg} / \mathrm{kg} \mathrm{NC}-6004$ or $2.5 \mathrm{mg} / \mathrm{kg}$ CDDP plus $10 \mathrm{mg} / \mathrm{kg} \mathrm{S}-1$. NC-6004, CDDP and S-1 were administered according to the abovementioned schedules. In further analyses, enhancement of the antitumor effect was evaluated following dose escalation of combination therapies as follows: Mice from the combination therapy groups were treated with $10 \mathrm{mg} / \mathrm{kg} \mathrm{S}-1$ plus 5.0 or $7.5 \mathrm{mg} / \mathrm{kg}$ NC-6004. Combination S-1/CDDP therapy was administered as follows: $10 \mathrm{mg} / \mathrm{kg}$ S-1 plus 5.0 or $7.5-\mathrm{mg} / \mathrm{kg}$ CDDP. NC-6004 and CDDP were 
- $5-\mathrm{FU}+\mathrm{CDDP}$
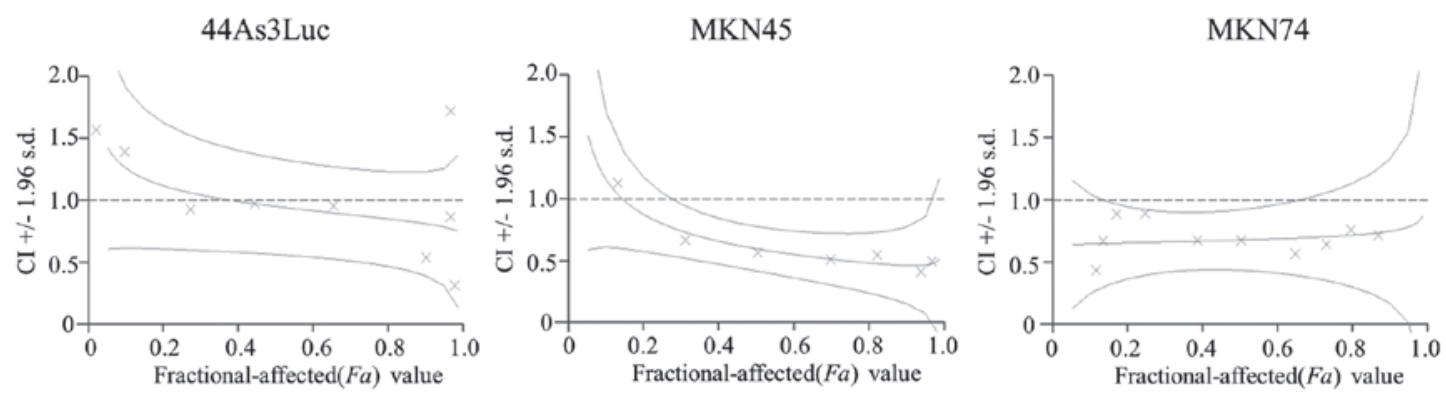

- $5-\mathrm{FU}+\mathrm{NC}-6004$
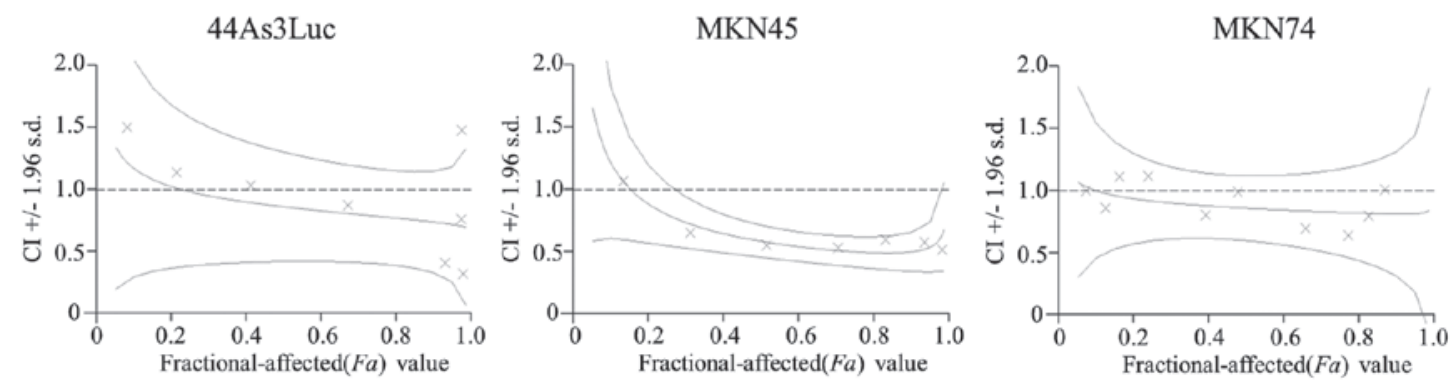

Figure 1. Combination indices (CI) of 5-FU with CDDP or NC-6004. A CI of $<1.0,1.0$ and $>1.0$ is indicative of synergistic, additive and antagonistic interactions, respectively. 5-FU, 5-fluorouracil; CDDP, cisplatin; SD, standard deviation.

administered on days 1,8 and 15 , whereas $\mathrm{S}-1$ was administered on days $0-4,7-11$ and 14-18. Treatment was discontinued when the mean body weight decreased $>10 \%$ from the baseline.

Evaluation of nephrotoxicity and pathological examination of the small intestine. Female BALB/c nude mice were divided into four groups of 4-6 mice each (four for control group and six for treatment group): The S-1/NC-6004 group, the S-1/CDDP group, the S-1 group and the control group. NC-6004, CDDP and S-1 were administered according to the dosing schedules described above, using $10 \mathrm{mg} / \mathrm{kg} \mathrm{S}-1$ plus $7.5 \mathrm{mg} / \mathrm{kg} \mathrm{NC}-6004$ or $10 \mathrm{mg} / \mathrm{kg} \mathrm{S}-1$ plus $7.5 \mathrm{mg} / \mathrm{kg}$ CDDP. In this experiment, treatment was continued even when the mean body weight decreased $>10 \%$ from the baseline. On day 21 , the mice were sacrificed under general anesthesia, blood samples were collected, and the small intestine was removed for further investigation. The creatinine levels in the blood samples were determined by SRL Laboratories (Tokyo, Japan). The small intestine was excised at the middle portion of the ileum, fixed in $10 \%$ formalin, embedded in paraffin, sectioned and stained with hematoxylin and eosin.

Statistical analysis. All data are expressed as mean \pm standard deviation (SD). Tumor growth inhibition effect, body weight changes and renal function (serum creatinine level) were compared using Dunnett's two-tailed $t$-tests. Statistical analyses were performed using JMP 10 (SAS Institute, Inc., Cary, NC, USA) and differences were considered statistically significant when $\mathrm{P}<0.05$.

\section{Results}

In vitro growth inhibition assays. The $\mathrm{IC}_{50}$ values of $\mathrm{NC}-6004$ in gastric cancer cell lines ranged from 21.75 (44As3Luc) to
Table I. $\mathrm{IC}_{50}$ values $(\mu \mathrm{mol} / \mathrm{l})$ of CDDP and NC-6004 in the 44As3Luc, MKN45 and MKN74 gastric cancer cell lines.

\begin{tabular}{lcrr}
\hline \multirow{2}{*}{ Cell line } & \multicolumn{2}{c}{ IC50 $(\mu \mathrm{mol} / \mathrm{l}) \pm$ standard deviation } \\
\cline { 2 - 4 } & \multicolumn{1}{c}{5 -FU } & \multicolumn{1}{c}{ CDDP } & \multicolumn{1}{c}{ NC-6004 } \\
\hline 44As3Luc & $3.90 \pm 0.15$ & $1.94 \pm 0.20$ & $21.75 \pm 0.76$ \\
MKN45 & $5.69 \pm 0.50$ & $9.52 \pm 1.32$ & $133.13 \pm 4.93$ \\
MKN74 & $3.21 \pm 0.39$ & $19.38 \pm 1.15$ & $118.13 \pm 9.15$ \\
\hline
\end{tabular}

$\mathrm{IC}_{50}, 50 \%$ inhibitory concentration; 5-FU, 5-fluorouracil; CDDP, cisplatin.

$118.13 \mu \mathrm{mol} / 1$ (MKN74; Table I). In agreement with a previous study (8), the cytotoxic effect of NC-6004 was between 6.1-and 14.0-fold lower compared with that of CDDP. Hence, molar ratios of 1:1 and 1:10 were used for the 5-FU:CDDP and 5-FU:NC-6004 combination studies, respectively.

Drug interactions between 5-FU and CDDP or NC-6004. CIs of $<1.0,1.0$ and $>1.0$ are indicative of synergistic, additive and antagonistic interactions between two agents, respectively. In all three cell lines, synergy was observed at Fa values 0.5-1.0 for both combination therapies (Fig. 1), and the synergy between 5-FU and NC-6004 was almost equivalent to the synergy between 5-FU and CDDP in 44As3Luc cells.

In vivo antitumor effects of single agents and combinations in 44As3Luc tumors. The antitumor effects and body weight loss following administration of NC-6004, CDDP and combinations of $10 \mathrm{mg} / \mathrm{kg} \mathrm{S}-1$ plus $2.5 \mathrm{mg} / \mathrm{kg}$ CDDP or NC-6004 were similar with the antitumor effects and body weight 

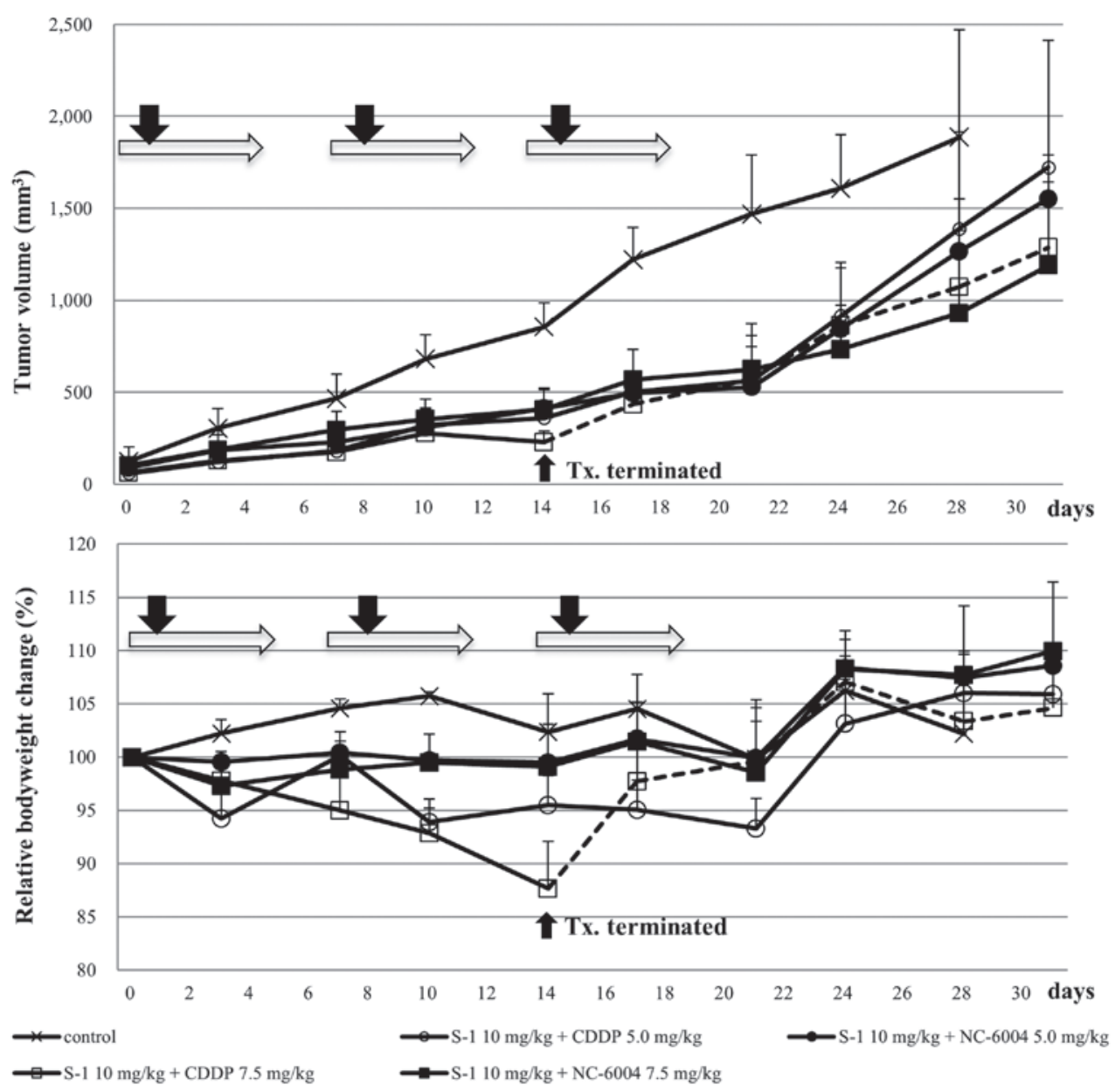

Figure 2. In vivo tumor growth inhibition assays of combination therapies in mice ( $\mathrm{n}=6)$. Combined treatment with $10 \mathrm{mg} / \mathrm{kg} \mathrm{S}-1$ on days $0-4,7-11$ and $14-18$ plus $7.5 \mathrm{mg} / \mathrm{kg}$ CDDP or NC-6004 on days 1,8 and 15 . Combined treatment with $10 \mathrm{mg} / \mathrm{kg} \mathrm{S}-1$ plus $7.5 \mathrm{mg} / \mathrm{kg}$ CDDP was discontinued on day 14 due to body weight loss of $>10 \%$. Average body weights were recovered subsequently (dotted line). Tx, treatment; CDDP, cisplatin.

A

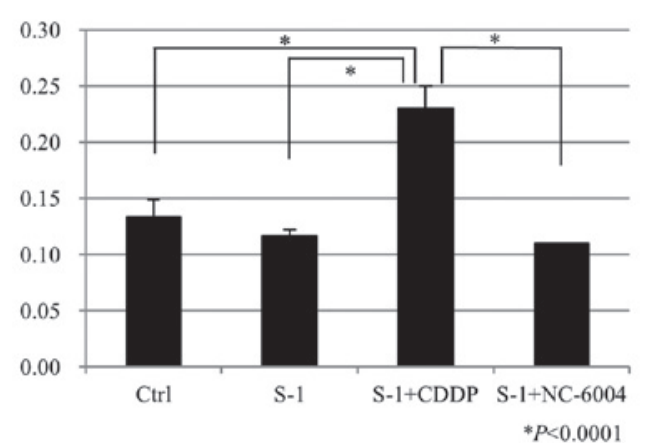

B

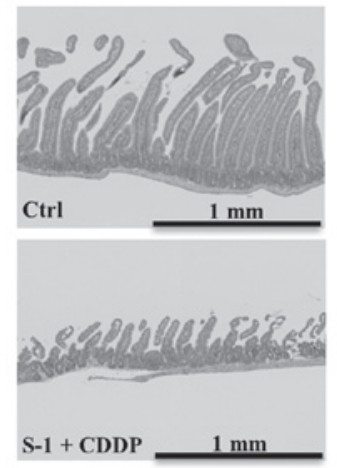

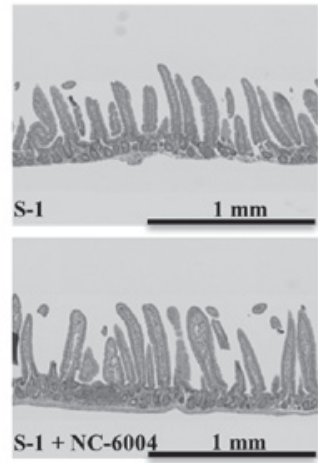

Figure 3. Evaluation of renal and intestinal toxicity of S-1 plus CDDP or NC-6004. (A) Plasma creatinine concentrations on day 21; (B) Histopathological findings in the ileal mucosa. The mucosa displayed several pathological changes due to the toxicity of the combination therapy, including deformed villi and decreased villus density. Ctrl, control; CDDP, cisplatin.

loss following administration of NC-6004 and CDDP, and both factors increased in a dose-dependent manner (data not shown). Combination treatment with S-1 and $7.5 \mathrm{mg} / \mathrm{kg}$ CDDP was discontinued on day 14 due to body weight losses of $>10 \%$ from the baseline. However, the therapeutic effect of S-1 plus $5.0 \mathrm{mg} / \mathrm{kg}$ NC-6004 did not differ significantly from the effect of S-1 and $5.0 \mathrm{mg} / \mathrm{kg}$ CDDP. Similarly, the therapeutic effect of S-1 plus $7.5 \mathrm{mg} / \mathrm{kg} \mathrm{NC}-6004$ did not differ significantly from the effect of S-1 plus $5.0 \mathrm{mg} / \mathrm{kg} \mathrm{NC}-6004$ or CDDP until day 21 , although a non-significant trend toward greater efficacy of S-1 plus $7.5 \mathrm{mg} / \mathrm{kg}$ NC- 6004 was observed, starting on day 21 . By contrast, the body weight changes were significantly more prominent in the group treated with $\mathrm{S}-1$ plus $5.0 \mathrm{mg} / \mathrm{kg}$ CDDP compared with those in the group treated with S-1 plus 
$5.0 \mathrm{mg} / \mathrm{kg}$ NC-6004. There were no significant differences in body weight loss between the S-1 plus $5.0 \mathrm{mg} / \mathrm{kg} \mathrm{NC}-6004$ and $\mathrm{S}-1$ plus $7.5 \mathrm{mg} / \mathrm{kg} \mathrm{NC}-6004$ groups. However, the combination of S-1 plus $7.5 \mathrm{mg} / \mathrm{kg}$ CDDP led to severe body weight loss compared with all the other groups.

Nephrotoxicityand intestinal toxicity of combination therapies. The plasma concentrations of creatinine \pm SD on day 21 after administration of $0.9 \% \mathrm{NaCl}, \mathrm{S}-1, \mathrm{~S}-1 / \mathrm{CDDP}$, or S-1/NC-6004 were $0.13 \pm 0.02,0.12 \pm 0.01,0.23 \pm 0.02$ and $0.11 \pm 0.00 \mathrm{mg} / \mathrm{dl}$, respectively. The $\mathrm{S}-1$ plus $7.5 \mathrm{mg} / \mathrm{kg}$ CDDP group had significantly higher creatinine plasma concentrations compared with the control group $(\mathrm{P}<0.0001)$, the $\mathrm{S}-1$ group $(\mathrm{P}<0.0001)$ and the $\mathrm{S}-1$ plus NC-6004 group $(\mathrm{P}<0.0001$; Fig. 3A).

Regular alignment of normal villi in the jejunal and ileal mucosa from mice treated with the $0.9 \% \mathrm{NaCl}$ solution was observed. By contrast, the jejunal and ileal mucosa from mice in the S-1, S-1/CDDP and S-1/NC-6004 treatment groups displayed deformed villi with decreased villus height and width. In particular, mice treated with S-1/CDDP exhibited more severe deformations and changes in villus size, and more severe decreases in villus density compared with mice treated with S-1 and S-1/NC-6004 (Fig. 3B).

\section{Discussion}

In the present study, the antitumor activity and the toxicity of S-1/NC-6004 combination therapy were compared to those of S-1/CDDP combination therapy, which is one of the most common treatments currently used for gastric cancer. Our data indicate that $\mathrm{S}-1 / \mathrm{NC}-6004$ therapy is significantly less toxic compared with S-1/CDDP therapy. Moreover, the antitumor activity of S-1/NC-6004 did not differ significantly from the antitumor activity of S-1/CDDP in the mouse model of human gastric cancer used in this study. Furthermore, when administered in combination with $\mathrm{S}-1$, the antitumor effects of NC-6004 and CDDP increased in a dose-dependent manner compared with the effects of treatment with a single therapeutic agent. However, the toxicity of CDDP also increased in a dose-dependent manner, necessitating the cessation of treatment with $10 \mathrm{mg} / \mathrm{kg} \mathrm{S}-1$ plus $7.5 \mathrm{mg} / \mathrm{kg}$ CDDP. By contrast, the toxicity of NC-6004 did not increase in a dose-dependent manner, and combined treatment with $10 \mathrm{mg} / \mathrm{kg} \mathrm{S}-1$ and $7.5 \mathrm{mg} / \mathrm{kg}$ NC-6004 was well-tolerated. Furthermore, the combination of S-1 and NC-6004 did not cause nephrotoxicity. It was previously suggested that S-1 dosing should be determined based on renal function, as excessive concentrations of $\mathrm{S}-1$ in the blood cause severe adverse effects, including bone marrow suppression and GI toxicity (13). Therefore renal function must be closely monitored during combination treatment with S-1 and CDDP. By contrast, NC-6004 is less likely to cause renal dysfunction compared with CDDP, and myelosuppression and GI toxicity may be comparatively limited following combination therapy with $\mathrm{S}-1$. Although we did not measure blood concentrations of the tegafur/S-1 metabolite 5-FU, our data suggest that elevated concentrations of S-1 in the blood contributed to GI toxicity due to CDDP-induced renal function impairment.

In the phase I clinical trial of NC-6004 (11), the recommended dose was defined as the equivalent of a $90 \mathrm{mg} / \mathrm{m}^{2}$ dose of CDDP. This trial also demonstrated that excessive hydration is not required during administration of NC-6004, allowing safe administration on an outpatient basis. Accordingly, a phase III clinical trial in pancreatic cancer and a phase II trial in non-small-cell lung cancer are currently underway. Combination chemotherapy with CDDP has been used as a standard treatment regimen for gastric cancer as well as several other types of cancer. Replacing CDDP with NC-6004 may lead to reduced toxicity of these treatment regimens and improve the quality of life of cancer patients.

In conclusion, the present preclinical study demonstrated that combined treatment with S-1 and NC-6004 is associated with significantly lower toxicity compared with S-1 and CDDP, while retaining similar CDDP-mediated antitumor activity. These data warrant further clinical studies of the combination of S-1 and NC-6004 in patients with advanced gastric cancer.

\section{Acknowledgements}

The present study was supported by the National Cancer Center Research and Development Fund (Y. Matsumura) and by the Japan Society for the Promotion of Science (JSPS), through the 'Funding Program for World-Leading Innovative R\&D on Science and Technology' (FIRST). The authors would like to thank Mr. Akifumi Furuya and Ms. Mari Mizoguchi for their technical assistance, and Ms. Madoka Nakayama for the secretarial assistance.

\section{References}

1. Koizumi W, Narahara H, Hara T, Takagane A, Akiya T, Takagi M, Miyashita K, Nishizaki T, Kobayashi O, Takiyama W, et al: S-1 plus cisplatin versus $\mathrm{S}-1$ alone for first-line treatment of advanced gastric cancer (SPIRITS trial): A phase III trial. Lancet Oncol 9: 215-221, 2008.

2. Pinzani V, Bressolle F, Haug IJ, Galtier M, Blayac JP and Balmès P: Cisplatin-induced renal toxicity and toxicity-modulating strategies: A review. Cancer Chemother Pharmacol 35: 1-9, 1994.

3. Hartmann JT and Lipp HP: Toxicity of platinum compounds. Expert Opin Pharmacother 4: 889-901, 2003.

4. Yamada Y, Higuchi K, Nishikawa K, Gotoh M, Fuse N, Sugimoto N, Nishina T, Amagai K, Chin K, Niwa Y, et al: Phase III study comparing oxaliplatin plus S-1 with cisplatin plus S-1 in chemotherapy-naïve patients with advanced gastric cancer. Ann Oncol 26: 141-148, 2015.

5. Matsumura Y and Maeda H: A new concept for macromolecular therapeutics in cancer chemotherapy: Mechanism of tumoritropic accumulation of proteins and the antitumor agent smancs. Cancer Res 46: 6387-6392, 1986.

6. Maeda $\mathrm{H}$ and Matsumura Y: EPR effect based drug design and clinical outlook for enhanced cancer chemotherapy. Adv Drug Deliv Rev 63: 129-130, 2011.

7. Nishiyama $\mathrm{N}$ and Kataoka K: Preparation and characterization of size-controlled polymeric micelle containing cis-dichlorodiamm ineplatinum(II) in the core. J Control Release 74: 83-94, 2001.

8. Uchino H, Matsumura Y, Negishi T, Koizumi F, Hayashi T, Honda T, Nishiyama N, Kataoka K, Naito S and Kakizoe T: Cisplatin-incorporating polymeric micelles (NC-6004) can reduce nephrotoxicity and neurotoxicity of cisplatin in rats. Br J Cancer 93: 678-687, 2005.

9. Levi F, Hrushesky WJ, Borch RF, Pleasants ME, Kennedy BJ and Halberg F: Cisplatin urinary pharmacokinetics and nephrotoxicity: A common circadian mechanism. Cancer Treat Rep 66: 1933-1938, 1982.

10. Mizumura Y, Matsumura $\mathrm{Y}$, Hamaguchi T, Nishiyama $\mathrm{N}$, Kataoka K, Kawaguchi T, Hrushesky WJ, Moriyasu F and Kakizoe T: Cisplatin-incorporated polymeric micelles eliminate nephrotoxicity, while maintaining antitumor activity. Jpn J Cancer Res 92: 328-336, 2001. 
11. Plummer R, Wilson RH, Calvert H, Boddy AV, Griffin M, Sludden J, Tilby MJ, Eatock M, Pearson DG, Ottley CJ, et al: A Phase I clinical study of cisplatin-incorporated polymeric micelles (NC-6004) in patients with solid tumours. Br J Cancer 104: 593-598, 2011.

12. Yanagihara K, Takigahira M, Takeshita F, Komatsu T, Nishio K, Hasegawa $\mathrm{F}$ and Ochiya T: A photon counting technique for quantitatively evaluating progression of peritoneal tumor dissemination. Cancer Res 66: 7532-7539, 2006.

13. Chou TC and Talalay P: Quantitative analysis of dose-effect relationships: The combined effects of multiple drugs or enzyme inhibitors. Adv Enzyme Regul 22: 27-55, 1984.
14. Booka E, Imamura CK, Takeuchi $H$, Hamamoto $Y$ Gomi D, Mizukami T, Ichiyama T, Tateishi K, Takahashi T, Kawakubo H, et al: Development of an S-1 dosage formula based on renal function by a prospective pharmacokinetic study. Gastric Cancer 19: 876-886, 2016. 